\title{
Design of Model-based Controller with Disturbance Estimation in Steer- by-wire System
}

\author{
Sanghun Jung ${ }^{1}$, Seibum Choi ${ }^{1, a}$ \\ ${ }^{1,1 a}$ KAIST, Department of Mechanical Engineering, 34141 Daejeon, Republic of Korea
}

\begin{abstract}
The steer-by-wire system is a next generation steering control technology that has been actively studied because it has many advantages such as fast response, space efficiency due to removal of redundant mechanical elements, and high connectivity with vehicle chassis control, such as active steering. Steer-by-wire system has disturbance composed of tire friction torque and self-aligning torque. These disturbances vary widely due to the weight or friction coefficient change. Therefore, disturbance compensation logic is strongly required to obtain desired performance. This paper proposes model-based controller with disturbance compensation to achieve the robust control performance. Targeted steer-by-wire system is identified through the experiment and system identification method. Moreover, model-based controller is designed using the identified plant model. Disturbance of targeted steerby-wire is estimated using disturbance observer(DOB), and compensate the estimated disturbance into control input. Experiment of various scenarios are conducted to validate the robust performance of proposed model-based controller.
\end{abstract}

\section{Introduction}

$\mathrm{X}$-by-wire (XBW) system is being introduced in the future automotive as the next generation automotive control system [1]. XBW system is being developed to provide the driver with the convenience of driving, steering, and braking. With this trend, Steer-by-wire (SBW) system is a future-oriented next-generation steering system with a large attention in the automotive industry. In SBW system, mechanical and hydraulic elements are removed. And electrical signal and actuator is used to control the steering system. Therefore, a driver and a wheel physically separated completely.

There are two main issues in SBW system control. First issue is applying reaction torque to the driver to improve the 'steering feel'. Due to the separation between driver and wheel, driver cannot feel the road information from wheel. Therefore, many studies are undergoing to apply reaction torque to compensate the 'steering feel'. Second issue is obtaining high control performance. Due to the separation, SBW system must have high tracking control performance.

In previous works, developing SBW control system is mainly focused on first issue ([2], [3], and [4]). Most of papers use simple PID controller as lower level controller to obtain the tracking performance. In the paper that proposes a controller that responds more strongly to disturbances, friction torque and self-aligning moment are modeled and added as feedforward term. However, this paper assumes that friction coefficient between road and wheel and vehicle body weight is constant. However, on vehicles with a light weight, such as micro-mobility, weight changes in wide range depending on the number of passengers. In addition, friction coefficient between road and wheel varies wide range with the road condition [5]. As a result, friction torque and self-aligning moment varies in wide range. Because of this variation of disturbance torques, constant feedforward term will not compensate enough for disturbance.

This paper proposes the robust lower level controller for SBW system. Targeted SBW system is modeled and identified through experiment results. Identified model is used to construct model-based controller. By using the model-based controller, we can achieve the fast response in transient input. Also, the friction torque and the selfaligning moment varying in a large range are treated as system disturbances. And we construct the disturbance observer (DOB) to estimate the system disturbances and compensate it to the control input. Thus, disturbance is rejected and we can achieve the robust control for various scenario. Proposed controller is validated through experiment using SBW system of 3-wheeler micromobility. Experiments are carried out according to the three road conditions that indicate friction torque varies. Results show that friction torque is well estimated, and performance of proposed controller is guaranteed regardless of road condition.

The rest of this paper is organized as follows. Section 2 presents the system modeling and identification method. Experimental setup, identification results are presented in this section. Section 3 presents the model-based controller with disturbance estimation design. Experimental result of proposed controller is presents in section 4. Conclusion is provided in section 5 .

\footnotetext{
a Corresponding author: sbchoi@kaist.ac.kr
} 


\section{Steer-by-wire System Modeling \& Identification}

\subsection{System description}

In this paper, SBW system of light weigh 3-wheeler micro mobility is targeted. Targeted vehicle operated by 2 front in-wheel motors, and 1 rear steering motor.

To analyse and validate the proposed controller, we construct the test bench as Figure 1. Overall control algorithm is constructed using Matlab \& Simulink. Constructed controller is built in to the MicroAutoBox to generate the control input and receive the measurement data.

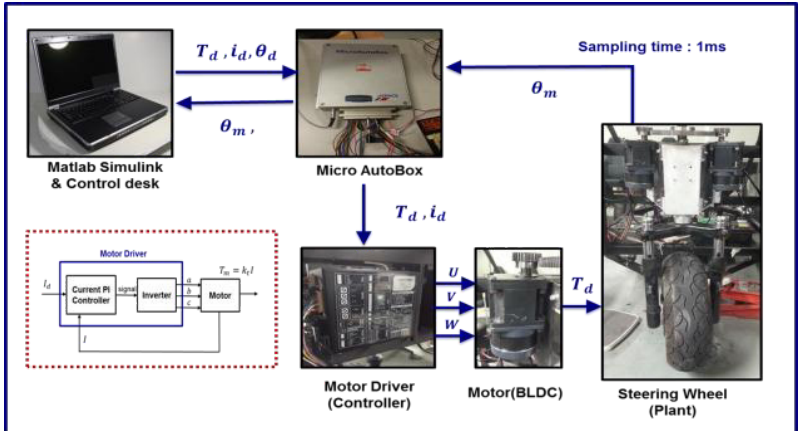

Figure 1. SBW system hardware and test bench set up

\subsection{System modeling \& identification}

Mechanical system of SBW can modeled as simple $2^{\text {nd }}$ order mass-damper system ([6], [7]). Two BLDC motors drive this system. Torque form motors is transferred through gearbox and rotate the steering wheel. So, we can summarize the system dynamics equation as following equivalent equation.

$$
I_{e q} \ddot{\theta}_{s}+B_{e q} \dot{\theta}_{s}+T_{\text {dist }}=2 N T_{m}=u
$$

Where

$$
\begin{aligned}
& I_{e q}=I_{s}+2 N^{2} I_{m} \\
& B_{e q}=B_{s}+2 N^{2} B_{m}
\end{aligned}
$$

If we ignore the disturbance torque from ground, i.e. $T_{\text {dist }}=0$, we can calculate the transfer function betweetn control input torque $T$ and steering wheel angle $\theta$ as follow.

$$
G(s)=\frac{\Theta(s)}{T(s)}=\frac{1}{I_{e q} s^{2}+B_{e q} s}
$$

In this research, we construct the model-based controller. Therefore, it is important to know the exact model information such as parameters or bandwidth. In the equation (4), system parameters are unknown. Therefore, we use the closed-loop system identification method to estimate the system parameters. The closed loop system transfer function is calculated as follow.

$$
\frac{\Theta(s)}{\Theta_{d}(s)}=\frac{K G(S)}{1+K G(S)}=\frac{K}{I_{e q} s^{2}+B_{e q} s+K}
$$

Where $\theta_{d}$ is commanded steering angle, $\theta$ is actual steering angle, and $K$ is the feedback gain of closed loop system. To analyze the frequency response of the system, we apply 0 to $5 \mathrm{~Hz}$ sine wave as command input. Figure 2 show the command input and following results.

Through frequency response of the system, we use the empirical transfer function estimate(ETFE) to estimate the transfer function. Using the discrete fourier transform (DFT), we compare the ratio phase between commanded input and acutal ouput angle. As a results, we can calculate the bode plot form experiment data and use it to the system identification. Figure 3 shows the result of ETFE. We apply the command input in 0 to $5 \mathrm{~Hz}$, so we do not use the information over $5 \mathrm{~Hz}$ range of calculated bode plot. Finally, we can obtain system parameters $I_{e q}$ and $B_{e q}$.

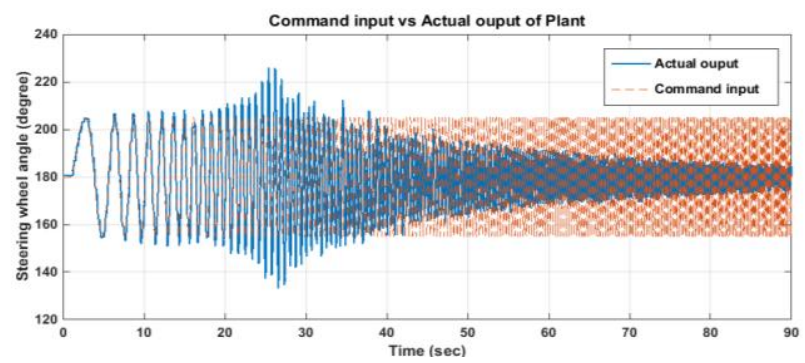

Figure 2. Experiment result: Command and actual angle for system identification.
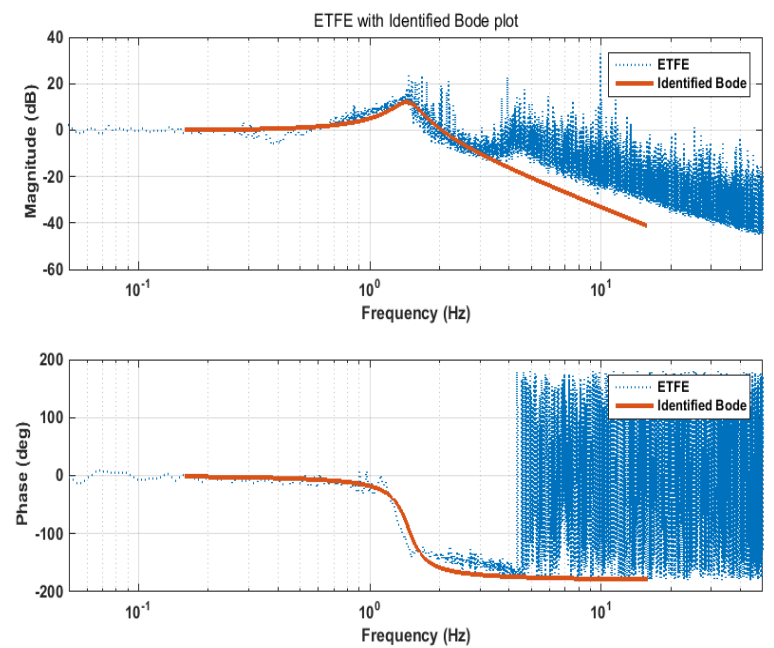

Figure 3. ETFE with identified bode plot

\section{Model-based controller design with disturbance estimation}

\subsection{Model-based controller design}

In previous section, targeted SBW system is modeled in $2^{\text {nd }}$ order system and well identified. Therefore, we can construct the model-based controller using identified model. 
Control target of the system is track the real steering wheel angle $\theta$ to desired command angle $\theta_{d}$. Thus, we can set the error as following.

$$
e=\theta_{d}-\theta
$$

Fianl control target is to make the error equation converge to zero as time passed, error dynamic is constructed as folloing equation.

$$
\ddot{e}+3 \lambda \dot{e}+3 \lambda^{2} e+\lambda^{3} \int e=0
$$

The order of dynamic equation of the system is 2 as equation (1) shows. Generally, the order of error dynamics follows the system order. But, in this research, targeted system has a feature of wide range of large friction. Generally, friction in $2^{\text {nd }}$ order system causes the steady-state error. So, we apply the integral term to the $2^{\text {nd }}$ order error dynamic so that we can handle the steadystate error from friction. Furthermore, we place the pole of gains in single point, we can tune the gain more conveniently.

Combining system dynamic equation (1) and error dynamics (7), we can organize the equations as follow. $\ddot{e}=\ddot{\theta}_{d}-\ddot{\theta}=\ddot{\theta}_{d}-\frac{1}{I_{e q}}\left(u-B_{e q} \dot{\theta}-T_{\text {dist }}\right)=-3 \lambda \dot{e}-\beta \lambda^{2} e-\lambda^{3} \int e$

The above equation can be summarized in terms of $u$ as follow.

$$
u=I_{e q} \ddot{\theta}_{d}+B_{e q} \dot{\theta}+T_{\text {dsst }}+3 \lambda^{2} I_{e q} e+3 \lambda I_{e q} \dot{e}+\lambda^{3} I_{e q} \int e(9)
$$

In above equation, first part of input is feed-forward control based on identified model. And $T_{\text {dist }}$ is unknown disturbance from ground, and rest term is formed of PID feedback controller.

Model-based feed-forward controller calculate the control input from desired command signal immediately, it could obtain response that is much more fast rather than feedback control only. If the model is not accurate, system can be unstable. However, as shown in previous chapter, we assume that system is well modeled and well identified. Therefore, we proposed the model-based controller.

\subsection{Disturbance estimation using disturbance observer}

In previous section, model-based controller is constructed. However, the disturbance $T_{\text {dist }}$ is still unknown factor. As mentioned before, disturbance of SBW system is dominant factor of control. Thus, disturbance compensation logic is strongly requried. In this paper, we use the disturbance observer(DOB) to estimate and compensate the unknown disturbances.

The simple DOB structure of single input - single output(SISO) system is shown in Figure 4[8]. The notation $P, P_{n}$ indicate transfer functino of real plant and nominal plant respectively.

The final output $y$ can represented as function of reference input $r$ and disturbance $d$ as follow.

$$
Y(s)=T_{y r}(S) Y_{r}(s)+T_{y d}(s) D(s)
$$

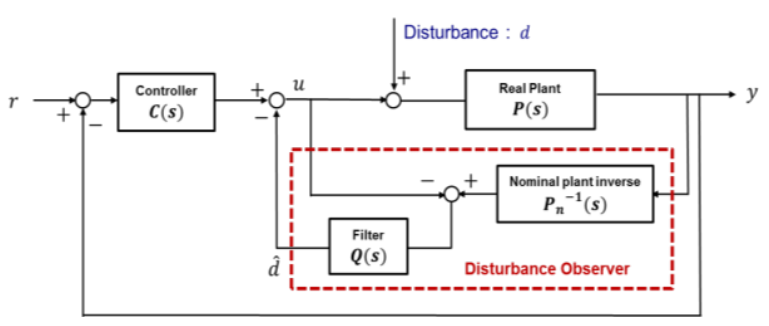

Figure 4. Basic structure and block diagram of DOB

$T_{r y}, T_{d y}$ is closed loop transfer function betweeen $r, d$ and output $y$ respectively. We can conclude that if Q-filter $Q(s)$ is well designed, we can acheive folloing results for low frequency range input under cutoff frequency $\omega_{q}$

$$
T_{r y}(s)=\frac{P_{n}(s) C(s)}{1+P_{n}(s) C(s)}, T_{d y}(s)=0
$$

This result means that SISO system with disturbance behaves same as nominal plant SISO system without disturbance. So disturbance is rejected internally by disturbance observer.

Applying above result in targeted SBW system, we can construct following euqations.

$$
\begin{gathered}
I_{e q} \ddot{\theta}+B_{e q} \dot{\theta}+T_{d i s t}=u \\
I_{n} \ddot{\theta}+B_{n} \dot{\theta}=u+\hat{d}
\end{gathered}
$$

The estimated disturbancd $\hat{d}$ is solved by above simultaneous equations. The result is as follow.

$$
\hat{d}=-\Delta I \ddot{\theta}-\Delta B \dot{\theta}-T_{\text {dist }}
$$

Where $\Delta I$ and $\Delta B$ represent the difference between real and nominal plant. If we assume that system is well identified so that $\Delta I=\Delta B=0$, estimated disturbance $\hat{d}$ converges to $-T_{\text {dist }}$. Thus, if we combine this result and constructed model-based controller in (9), final form of model-based controller is made as follow.

$$
u=I_{e q} \ddot{\theta}_{d}+B_{e q} \dot{\theta}-\hat{d}+3 \lambda^{2} I_{e q} e+3 \lambda I_{e q} \dot{e}+\lambda^{3} I_{e q} \int e
$$

Therefore, controller composed of model-based feed forward controller, disturbance compensations term, and feedback controller.

\section{Experimental results}

\subsection{Model-based controller design}

We conduct the experiment to validate the proposed model-based controller with disturbance estimation. To validate the robust performance of controller, we 
construct the three scenarios that represent the various friction torque-applying situation. The disturbances of SBW system composed of friction torque and selfaligning moment as mentioned before. However, the experiment only deals with stationary vehicle scenarios, which means that the disturbance is composed of friction uniquely. Friction torque between wheel and road can be analyzed as follow.

$$
T_{\text {fric }}=k \cdot \mu F_{z}
$$

The notation $k, \mu$, and $F_{z}$ represent the constant, friction coefficient, and vehicle vertical load respectively. Equation (20) implies that friction torque can varies by changing the firciton toruqe coefficient or vehicle vertical load. Thus, we construct the three scenario with three different vehicle vertical loads. Because vertical load is much more easier to measure and analyze rather than friction coefficient. Table 1 shows the scenario and situation that each scenario implies.

The case 1 refers to the normal driving situation. The case 2 refers to the system with higher disturbance in the case of high-mu surface or vehicle with heavy load situation. The case 3 refers to the system with lower disturbance in the case of low-mu surfaces, such as ice or snow road. The case 3 was carried out by lifting the steering wheel.

Table 1. Experiment scenarios and implying situation.

\begin{tabular}{|c|c|c|}
\hline No. & Measured $F_{z}$ & Case information \\
\hline 1 & $100 \mathrm{kgf}$ & Normal driving \\
\hline 2 & $145 \mathrm{kgf}$ & High-mu surface, loaded vehicle \\
\hline 3 & $0 \mathrm{kgf}$ & Low-mu surface: ice, snow road \\
\hline
\end{tabular}

It can be said that propsed controller has robust control performance if the controller performance guranteed through the above scenarios with single gain and without additional gain-scheduling.

The experiments are carried out acoording to the each scenario above applying the sinusoidal wave with $0.3 \mathrm{~Hz}$ and amplitude 30 degree. To confirm the effect of modelbased feedforward control and disturbance compensation logic, we conduct the experiment for each case with two differenct controller; (a) only feedback control, (b) model-based foeedforward control with disturbance estimation. Control input of each case is shown as follow.

$$
\begin{gathered}
u=3 \lambda^{2} I_{\text {oq }} e+3 \lambda I_{\text {oq }} \dot{e}+\lambda^{3} I_{\text {oq }} \int_{e} e \\
u=I_{\text {oq }} \ddot{\theta}_{\dot{\alpha}}+B_{\text {eq }} \dot{\theta}-\hat{d}+3 \lambda^{2} I_{\text {eq }} e+3 \lambda I_{\text {oq }} \dot{e}+\lambda^{3} I_{\text {oq }} \int_{e} e
\end{gathered}
$$

During the experiment, feedback gains are fixed. The experiment reuslts is shown as follows.
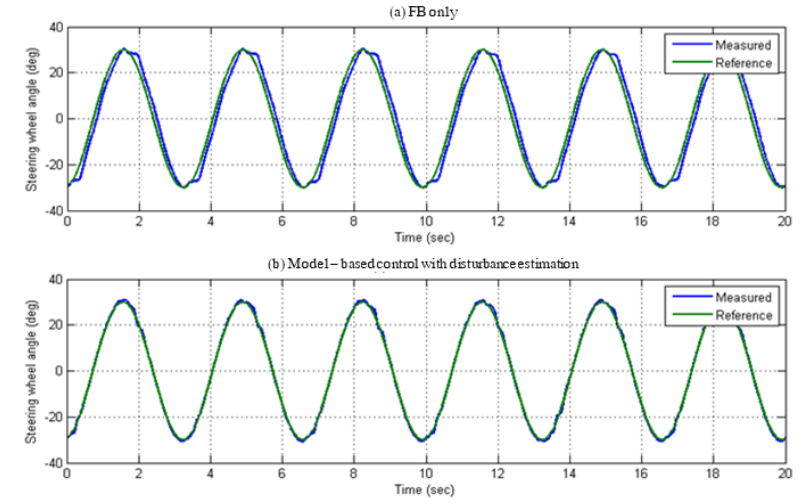

Figure 5. Experiment results: Scenario 1. Normal driving situation. (a) FB only (b) model-based control with disturbance estimation.

Experiment result for scenario 1 show the effect of model-based controller. Only feedback control, as shown in (a), output signal is lagged and suppressed due to the friction. Thus, we can show that reaction rate is lowered. However, as shown in (b), compensating the model-based feedforward term and disturbance estimation results make reaction rate much faster than (a). Therefore, tracking performance is improved.

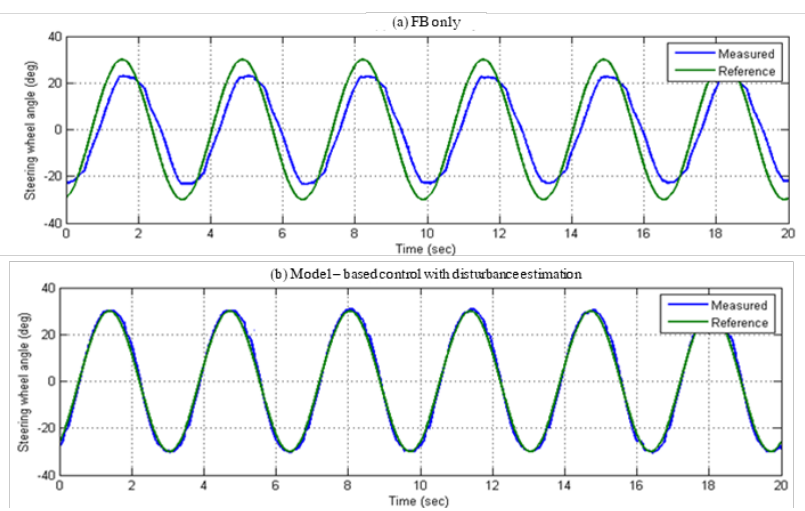

Figure 6. Experiment results: Scenario 2. High-mu surface or loaded vehicle. (a) FB only (b) model-based control with disturbance estimation.

The performance of the proposed controller is more prominent in scenario 2. This scenario implies larger friction, graph (a) shows the poor tracking performance. Due to the large friction, system output suppressed a lot and response rate becomes low. However, a term that compensates for the disturbance is included in the proposed controller. Thererfore, control ouput of proposed controller shows good perfermance with same feedback gains. 

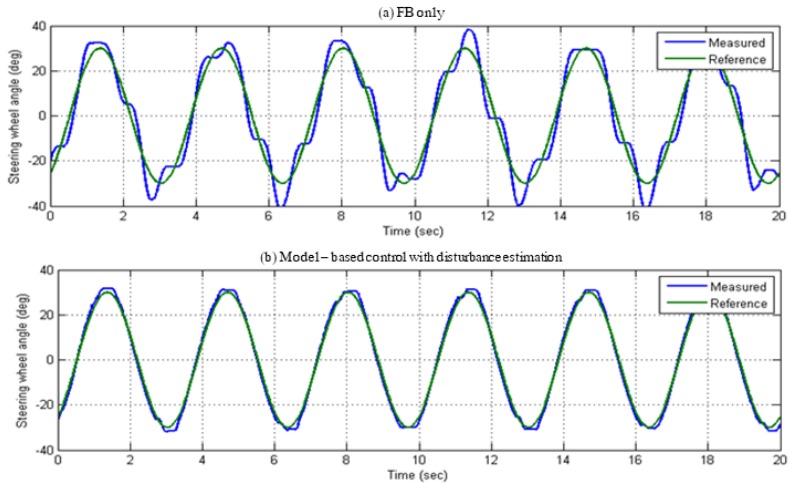

Figure 7. Experiment results: Scenario 3. Low-mu surface. (a) FB only (b) model-based control with disturbance estimation.

Scenario 3 indicates the frictionless system, which means a system without disturbances. Friction torque is the major part of the system as mentioned before. Thus, feedback gain is adjusted high-gain. However, if the friction disappears in that state, the systems becomes unstable as shown in (a). As mentioned in section 3.2, disturbance observer makes the system to behave as nominal plant. Therefore, if the gain is adjusted appropriately so that the nominal plant can operate in stable region, the proposed controller can obtain good tracking result. The result shown in (b).

As a results, propsed controller can obtain good tracking performance in various scenarios with fixed feedback gain. Therefore, we can conclude that proposed controller has robust control performance in SBW system. Table 2 shows the RMS error for each case of expriement.

Table 2. RMS error of proposed controller

\begin{tabular}{|c|c|c|}
\hline No. & Measured $F_{z}$ & RMS error (deg) \\
\hline 1 & $100 \mathrm{kgf}$ & 0.6924 \\
\hline 2 & $145 \mathrm{kgf}$ & 1.2338 \\
\hline 3 & $0 \mathrm{kgf}$ & 1.3248 \\
\hline
\end{tabular}

\section{Conclusion}

In light weight micro-mobility, disturbance is major part of the SBW system control. Therefore, disturbance compensation logic was strongly demanded. In this paper, we proposed the model-based controller with disturbance estimation in SBW system. Experiments are conducted to validate the proposed controller. Three scenarios representing the various disturbance are selected to validate the robustness of the proposed controller. As a result, model-based controller with disturbance estimation obtain robust control performance in various scenario with fixed feedback gain. The results of this research are expected to improve the performance of steering control of future-Micro-Mobility vehicle.

\section{Acknowledgment}

This work was supported in parts by the National Research Foundation of Korea grant funded by the Korea government (MSIP) (No.2017R1A2B4004116), the
Ministry of Science and ICT, Korea, under the Information Technology Research Center support program (IITP-2017-2012-0-00628) supervised by the Institute for Information and communications Technology Promotion, and the BK21 Plus Program.

\section{References}

1. Y.H.Ki, I.Oh, J.Jeon, J.Chun, H.S.Ahn, A new type of clamping force estimator for electromechanical brake systems, IEEE Transactions on Mechatronics, Vol.10, December, (2005)

2. Yung-Hsiang Judy Hsu, Estimation and Control of Lateral Tire Forces Using Steering Torque, Ph.D Dissertation, Stanford University, U.S, (2009)

3. Seok-Hwan Jang, Tong-Jin Parkl chang-soo Han, $A$ control of vehicle using steer-by-wire system with hardware-in-the-loop-simulation system, IEEE Transactions on Mechatronics, Vol.10, December, (2005)

4. Hiraku Ogura, Toshiyuki Murakami, Reaction Force Control on Accelerator Pedal and Steering Wheel based on Tire-Road Friction Forces, $35^{\text {th }}$ Annnual Conference of IEEE industrial Electronics, (2009)

5. Hiraku Ogura, Toshiyuki Murakami, Reaction Force Control on Accelerator Pedal and Steering Wheel based on Tire-Road Friction Forces, 35 ${ }^{\text {th }}$ Annnual Conference of IEEE industrial Electronics, (2009)

6. Sanket Amberkar, Farhad Bolourcho, A Control System Methodology for Steer by Wire Systems, SAE technical paper series, Steering and Suspension Technology Symposium (2004)

7. Paul Yih and J.Christian Gerdes, Modification of vehicle handling characteristics via Steer-by-Wire, IEEE Transactions on Control System Technology, Vol.13, No.6, November, (2005)

8. Shihua Li, Jun Yang, Wen-Hua chen, Xisong chen, "Disturbance Observer-Based Control, Methods and Applications", CRC Press, (2014) 